\title{
Polar Code Design for Nakagami-m Channel
}

\author{
Guo Rui $^{{ }^{*}}$ and Wu Yingjie ${ }^{1}$ \\ ${ }^{1}$ School of Communication Engineering, Hangzhou Dianzi University \\ Hangzhou, 310018, China \\ [e-mail: guorui@hdu.edu.cn] \\ *Corresponding author: Guo Rui
}

Received December 19, 2018; revised February 13, 2020; accepted June 6, 2020; published July 31, 2020

\begin{abstract}
One drawback of polar codes is that they are not universal, that is, to achieve optimal performance, different polar codes are required for different kinds of channel. This paper proposes a polar code construction scheme for Nakagami-m fading channel. The scheme fully considers the characteristics of Nakagami-m fading channel, and uses the optimized Bhattacharyya parameter bounds. The constructed code is applied to an orthogonal frequency division multiplexing (OFDM) system over Nakagami-m fading channel to prove the performance of polar code. Simulation result shows the proposed codes can get excellent bit error rate (BER) performance with successive cancellation list (SCL) decoding. For example, the designed polar code with cyclic redundancy check (CRC) aided SCL $(L=8)$ decoding achieves $1.1 \mathrm{~dB}$ of gain over LDPC at average BER about $10^{-5}$ under 4-quadrature amplitude modulation (4QAM) while the code length is 1024 , rate is 0.5 .
\end{abstract}

Keywords: Polar code, Bhattacharyya parameter bounds, Information bit selection, Nakagami-m fading, OFDM

This work was supported in part by the Zhejiang Provincial Natural Science Foundation of China under Grant LY16F010013 and in part by the National Natural Science Foundation Joint Research Program of China under Project U1709220. 


\section{Introduction}

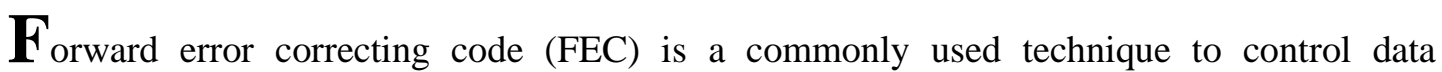
transmission errors in noisy channels [1], [2]. In 2009, Arikan proposed polar code, which is a new type of FEC. It has been theoretically proved to reach Shannon capacity over binary-input discrete memoryless channels (B-DMCs) as the code length tends to infinity [3]. Unlike the traditional capacity approximation codes such as Turbo and low density parity check (LDPC) codes, polar codes work on a different and unique methodology that is commonly known as "channel polarization" in the literature which synthesizes $N$ uncorrelated independent channels into a polarized channel with $N$ correlated polarized sub-channels. Polar codes have excellent performance, encoding and decoding complexities are much lower than other capacity-approaching coding schemes. Additionally, cyclic redundancy check (CRC) aided successive cancellation list (SCL) decoder for polar codes can get lower bit error rate even if the code length is short.

Polar code construction initially focused on simple discrete channels. But later polar code was extended to Rayleigh fading channel [4]. A more commonly encountered channel is the Nakagami-m channel, for which polar code is much less studied. This paper mainly studies polar codes construction over Nakagami-m channels and analyzes the bit error rate (BER) performances.

Because of the excellent characteristic against frequency selective fading, orthogonal frequency division multiplexing (OFDM) has very broad application prospect in wireless communications area [5][6]. And OFDM is often combined with channel coding to improve performance [7]. There exists extensive research on LDPC coded and Turbo coded OFDM systems, which have demonstrated excellent performance [8]-[11]. But most of these researches focus on additive white Gaussian noise (AWGN) channel.

Polar coded OFDM systems over Rayleigh fading channels and Rice fading channels have been studied in [12], [13]. For many communication scenarios, the Nakagami-m model fits better than the Rice model [14]. But to the authors' knowledge, polar coded OFDM system over Nakagami-m fading channel has rarely been studied. The first goal of this paper is to formulate the Bhattacharyya parameter for Nakagami-m fading channel. This allows us to construct polar codes with near optimum performance over Nakagami-m fading channel via optimizing the design of Bhattacharyya parameters. Then, polar coded OFDM system is studied, and also the performance of the derived polar code is compared with those of LDPC codes.

The structure of the rest paper is as follows. Section 2 introduces the construction scheme of polar codes over Nakagami-m fading channel. The system model of polar coded OFDM is simply presented in Section 3. In Section 4, the performance of the proposed information bit selection algorithm for polar codes over the Nakagami-m fading channel is evaluated, together with simulation results of polar coded OFDM systems over Nakagami-m fading channels.

\section{Polar Codes for Nakagami-m Channel}

\subsection{Channel Model}

Consider a received signal model

$$
y=h x+z
$$


where $x$ is the input signal, $y$ is the output signal, $z$ is the Gaussian noise, which satisfies the distribution of $z \sim N\left(0, \sigma^{2}\right)$ and $h$ is the channel gain satisfying Nakagami-m distribution $h \sim \frac{2 m^{m}}{\Gamma(m) \Omega^{m}} h^{2 m-1} \exp \left(-\frac{m}{\Omega} h^{2}\right)$ [15]. In this distribution, $\Gamma(m)$ represents the Gamma function, $m$ and $\Omega$ are two important parameters of Nakagami-m distribution: $\Omega$ is the spreading factor and $m(m \geq 1 / 2)$, is the shape factor, which is used to describe the fading severity. The cumulative distribution function (CDF) of the Nakagami-m distribution $F(h)$ is described as [16]:

$$
F(h)=\Gamma\left(m, \frac{m}{\Omega} h^{2}\right)
$$

Where $\Gamma(v, u)$ is the incomplete gamma function.

\subsection{Channel Polarization}

Arikan introduced the polarization process of B-DMC in reference [3]. Channel polarization is a technology that transforms $N$ identical and independent B-DMCs into $N$ correlated polarized channels. When $N$ approaches infinity, the polarized channels show polarization phenomenon, that is, these channels are either completely noiseless or completely noisy. The information transmission with Shannon capacity can be realized by choosing completely noiseless channel to transmit information bits. However, the code length $N$ of polar code is limited in practical application, so it is necessary to sort the channel according to the error rate of each polarized sub-channel and select the $K$ sub-channels with the best performance. Where $K$ means the number of information bit in polar code with code length $N$. However, the performance estimation of polarized sub-channel is challenging in practice, so many estimation schemes for different channels are proposed in references [17]-[22].

For a binary memoryless symmetric (BMS) channel, the transition probability is $W(x, y)$, $x, y \in(0,1)$, where $x$ represents the input bits and $y$ represents the output bits. Channel polarization is a kind of channel transformation. Through channel merging and decomposition, the original $N$ completely unrelated channels will be transformed into $N$ related channels $\left\{W_{N}^{(i)}: 1 \leq i \leq N\right\}$. Compared with the original channels, the total capacity of the channels has not changed after polarization, but the channel capacity of $W_{N}^{(i)}$ shows a polarization phenomenon: the channel capacity of $W_{N}^{(i)}$ is close to either 1 or 0 , as $N$ approaches infinity. The relationship between input bits $u_{1}^{N}$ and output bits $x_{1}^{N}$ of these polarized channels can be written as:

where $G_{N}$ is the generator matrix of size $N$.

$$
x_{1}^{N}=u_{1}^{N} G_{N}
$$

The polarized channels suggest a special channel coding scheme, the polar code. It achieves an excellent performance by transmitting $K$ information bits over $K$ channels whose symmetric capacity tends towards 0 and transmitting $N-K$ frozen bits over the rest channels whose symmetric capacity tends towards 1 .

\subsection{Construction of Polar Codes for Nakagami-m Channel}

Non-universality is an important characteristic of polar codes, that is, the construction scheme of polar codes is different for different channels. Most research about polar code are focuses on discrete channels, such as, BMC, binary erasure channel (BEC) and AWGN channel. A more practical and general communication channel, Rayleigh channel and Nakagami-m channel, has not been paid enough attention at present. This time, the construction and performance of polar codes over Nakagami-m channel are studied, which is a continuous and 
universal channel. Nakagami-m channel have different channel characteristic as compared with BEC, BMC, and AWGN channel. So, it is necessary to put forward different polar code construct algorithm. The polar encoder encodes the input bits $u_{1}^{N}$, and the encoded codes $x_{1}^{N}$ are obtained by using a simple transformation given in (3). The generator matrix $G_{N}$ is equal to $B_{N} F^{\otimes n}$ for any $N=2^{n}, n \geq 0$, where $B_{N}$ is a bit inversion matrix, $F=\left[\begin{array}{ll}1 & 0 \\ 1 & 1\end{array}\right]$, and $F^{\otimes n}$ represents the $n$-th Kronecker power of $F$, which is defined by $F^{\otimes n}=F \otimes F^{\otimes(n-1)}$.

The input bits $u_{1}^{N}$ is consisted of information bits and frozen bits. The frozen bits are determined for the receiver and do not need to be decoded. The most challenging part of polar encoding is finding information bit set A. Arikan proposed a method using Bhattacharyya parameter $Z(W)$ for B-DMC, which can be used to indicate the error probability [3]. The Bhattacharyya parameter is calculated as:

$$
Z(W)=\sum_{y} \sqrt{W(y / 0) W(y / 1)}
$$

where $W(y / 0)$ and $W(y / 1)$ are the transition probabilities of a channel when the input bits are 0 and 1 , respectively. It is clear that the smaller the Bhattacharyya parameter, the more reliable the channel is.

The Bhattacharyya parameters for the corresponding channels $\left\{W_{N}^{(i)}: 1 \leq i \leq N\right\}$ can be written as $\left\{Z^{(i)}: 1 \leq i \leq N\right\}$. $N-K$ channels with the largest Bhattacharyya parameter values in $\left\{Z^{(i)}: 1 \leq i \leq N\right\}$ transmit frozen bit, the rest channels transmit information bit. By this way, the polar codes with rate $R=K / N$ can be constructed.

The discussion mentioned above shows that key point of polar encoding is sub-channel selection. The formula of $Z(W)$ in (4) is only applicable to discrete channels. Nakagami-m fading channel is a continuous channel, which is an extension from the discrete channel, for which the Bhattacharyya parameter can be obtained by the following formula [23]:

$$
Z(W)=\int \sqrt{W(y / 0) W(y / 1)} d y
$$

Arikan proposed an iterative relation for BEC, which can be used to easily get the Bhattacharyya parameter of all polarized sub-channels easily [3]:

$$
\begin{gathered}
Z\left(W_{2 N}^{(2 i-1)}\right)=2 Z\left(W_{N}^{(i)}\right)-Z\left(W_{N}^{(i)}\right)^{2} \\
Z\left(W_{2 N}^{(2 i)}\right)=Z\left(W_{N}^{(i)}\right)^{2}
\end{gathered}
$$

For other channels, the Bhattacharyya parameters meet the following requirements:

$$
Z\left(W_{N}^{(i)}\right) \leq Z\left(W_{2 N}^{(2 i-1)}\right) \leq 2 Z\left(W_{N}^{(i)}\right)-Z\left(W_{N}^{(i)}\right)^{2}
$$

Based on these Bhattacharyya parameter bound, Bravo-Santos [23] presented the following three types of iterations:

Type 1:

$$
Z\left(W_{2 N}^{(2 i-1)}\right)=2 Z\left(W_{N}^{(i)}\right)-Z\left(W_{N}^{(i)}\right)^{2}
$$

Type 2:

$$
Z\left(W_{2 N}^{(2 i-1)}\right)=Z\left(W_{N}^{(i)}\right)
$$

Type 3: $\quad Z\left(W_{2 N}^{(2 i-1)}\right)=0.5 \times\left(2 Z\left(W_{N}^{(i)}\right)-Z\left(W_{N}^{(i)}\right)^{2}+Z\left(W_{N}^{(i)}\right)\right)$

In Nakagami-m fading channels, the initial value of the Bhattacharyya parameter is not fixed, but is related to the distribution function of the channel. The characteristics of Nakagami-m channel are described in Section 2.1. For a given input $x$, the transition probability of the channel is [24]:

$$
W(y \mid x)=\int_{0}^{\infty} W(y \mid h, x) f(h) d h
$$


where $W(y \mid h, x)$ is the conditional transition probability of the channel with known input and channel gain, and is determined by channel noise. $f(h)$ is the distribution function of channel gain. In Gaussian noise condition, we have

$$
W(y \mid x)=\int_{0}^{\infty} \frac{1}{\sqrt{2 \pi} \sigma} \exp \left(-\frac{(y-h x)^{2}}{2 \sigma^{2}}\right) \frac{2 m^{m}}{\Gamma(m) \Omega^{m}} h^{2 m-1} \exp \left(-\frac{m}{\Omega} h^{2}\right) d h
$$

which allows one to easily calculate $W(y \mid 0)$ and $W(y \mid 1)$ as:

$$
\begin{aligned}
& W(y \mid 0)=\Psi \int_{0}^{\infty} T \exp \left(-\frac{(y+h x)^{2}}{2 \sigma^{2}}\right) d h \\
& W(y \mid 1)=\Psi \int_{0}^{\infty} T \exp \left(-\frac{(y-h x)^{2}}{2 \sigma^{2}}\right) d h
\end{aligned}
$$

Where $\Psi=\frac{1}{\sqrt{2 \pi} \sigma} \frac{2 m^{m}}{\Gamma(m) \Omega^{m}}$ and $T=h^{2 m-1} \exp \left(-\frac{m}{\Omega} h^{2}\right)$. The above derivation shows that

$$
Z_{0}=\Psi \int_{-\infty}^{\infty} \sqrt{\int_{0}^{\infty} T \exp \left(-\frac{(y+h x)^{2}}{2 \sigma^{2}}\right) d h \int_{0}^{\infty} T \exp \left(-\frac{(y-h x)^{2}}{2 \sigma^{2}}\right) d h} d y
$$

\subsection{Polar Decoding}

Through the iterative operation, polar code decoder can figure out the estimated value of the information sequence according to the received sequence. Arikan proposed the successive cancellation (SC) decoder. Consider the source bit set $u_{1}^{N}=\left(u_{A}, u_{A^{c}}\right)$, where $u_{A}$ represents the information bits part and $u_{A^{c}}$ represents the frozen bits part. When the input sequence $u_{1}^{N}$ is transmitted across $W_{N}$, the output sequence $y_{1}^{N}$ can be obtained with the probability of $W_{N}\left(y_{1}^{N} \mid u_{1}^{N}\right)$. The SC decoder uses the sequence $\left(y_{1}^{N}, u_{A^{c}}\right)$ to calculate $\hat{u}_{1}^{N}$, the estimated value of $u_{1}^{N}$. The decoder obtains the decision $\hat{u}_{i}$ based on the likelihood ratio (LR) which is computed by using $y_{1}^{N}$ and the previous decisions $\hat{u}_{1}^{i-1}$ [23]:

where the decision function $h()$ is shown as follows

$$
\hat{u}_{i}=h\left(L_{N}^{(i)}\left(y_{1}^{N}, \hat{u}_{1}^{i-1}\right)\right)
$$

$$
h\left(L_{N}^{(i)}\left(y_{1}^{N}, \hat{u}_{1}^{i-1}\right)\right)=\left\{\begin{array}{lc}
0, & L_{N}^{(i)}\left(y_{1}^{N}, \hat{u}_{1}^{i-1}\right) \geq 1 \\
1, & \text { otherwise }
\end{array}\right.
$$

The initial value of the channel LR can be calculated by:

$$
L_{1}^{1}\left(y_{i}\right)=\frac{W\left(y_{i} \mid 0\right)}{W\left(y_{i} \mid 1\right)}
$$

However, for Nakagami-m fading channels, the calculation of channel LR also needs to be recalculated. Similar to the calculation of the initial value of Bhattacharyya parameter, from (14) and (15), we get the following function:

$$
L_{1}^{1}\left(y_{i}\right)= \begin{cases}\frac{\int_{0}^{\infty} T \exp \left(-\frac{(y+h)^{2}}{2 \sigma^{2}}\right) d h}{\int_{0}^{\infty} T \exp \left(-\frac{(y-h)^{2}}{2 \sigma^{2}}\right) d h}, & y_{i} \geq 0 \\ \frac{1}{L_{1}^{1}\left(\left|y_{i}\right|\right)}, & y_{i}<0\end{cases}
$$

Due to the limitation of sub-optimal approach via local search, SC decoder cannot find the optimal path in many cases. The SCL decoding algorithm can solve this problem. SCL decoder contains $L$ SC decoder components, where $L \geq 1$ is the list length, and the maximum value of the surviving path is $L$. This method can keep $L$ most likely code-words at each decoding step [25]. Therefore, the probability of finding the correct decoding path is significantly increased.

In [26], Niu and Chen proposed CRC-aided SCL decoding algorithm, which setup a rule of path choose by adding CRC verification information to the code-word . The BER of polar codes can be significantly reduced by CRC-aided SCL decoding even over Nakagami-m fading channels. 


\section{Polar Coded OFDM System}

The OFDM system with polar codes is dissected in Fig. 1.

At the transmitter, a serial high-speed data stream is encoded by polar codes. The coded data stream is then modulated by a digital modulator. After serial-to-parallel (S/P) conversion, the high speed data stream is transformed into a series of low speed parallel sub-stream. The longer symbol period reduces the inter-code interference caused by the channel multipath. The parallel data streams are then processed by an inverse fast Fourier transform (IFFT) block, followed by parallel-to-serial(P/S) conversion and insertion of cyclic prefix (CP), aiming to minimize inter-code and inter-carrier interference[27].

At the receiver, the frequency domain signal is obtained by a series of operations such as analog-to-digital (A/D) conversion, removal of the CP and fast Fourier transform (FFT). The demodulator then provides an estimate of the frequency domain signal. Finally, the signal is feed into the polar decoder.

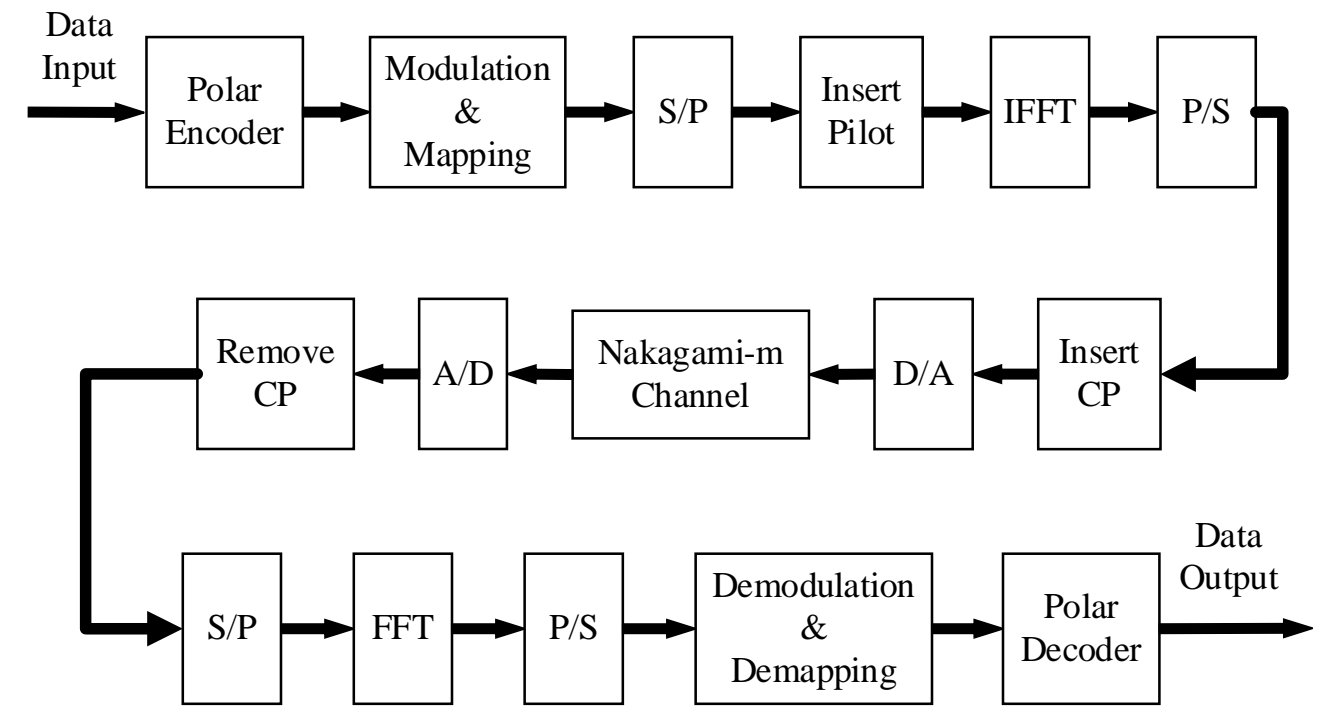

Fig. 1. The dissection of a OFDM system with polar codes

\section{Data analysis and Discussion}

In order to study the system proposed in this manuscript in detail, here we first analyze the influence of different fading coefficients of Nakagami-m fading channels on the system. The parameters adopted for the OFDM system are listed in Table 1.

Table 1. Parameter Settings for The Simulation

\begin{tabular}{cc}
\hline \hline Parameter & Value \\
\hline Number of subcarrier & 64 \\
Digital modulation technique & 4-Quadrature amplitude modulation (4QAM) \\
Noise channel model & Nakagami-m fading channel \\
Shape factor $(m)$ & $0.5,1,2,4$ \\
Spreading factor $(\Omega)$ & 1 \\
Signal frequency & $8 \mathrm{MHz}$ \\
Subcarrier frequency & $0.125 \mathrm{MHz}$ \\
SNR & $0-15 \mathrm{~dB}$ \\
\hline \hline
\end{tabular}


Fig. 2 shows the BER performance of OFDM system with 4QAM over Nakagami-m fading channels for different shape factors. It is observed that as the shape factor $\mathrm{m}$ increases, at the same SNR, the average BER decreases.

To assess the performance improvement with polar coding, the performances of OFDM system with and without channel coding are simulated and compared. LDPC codes and polar codes are selected as the channel coding scheme. And the modulation scheme is $4 \mathrm{QAM}$ for all. The method of directly assigning the initial value of Bhattacharyya parameter is used to construct the polar code, and CRC-SCL decoding algorithm is used. In order to get the Bhattacharyya parameter, $\Omega$ is set to 1 and variance $\sigma^{2}$ of Gaussian noise is set to 1 also. LDPC codes are constructed by progressive edge growth (PEG) algorithm without length-6 circle and decoded by BP decoder (16 iterations for LDPC codes). Parameters for polar codes and LDPC codes are listed in Table 2.

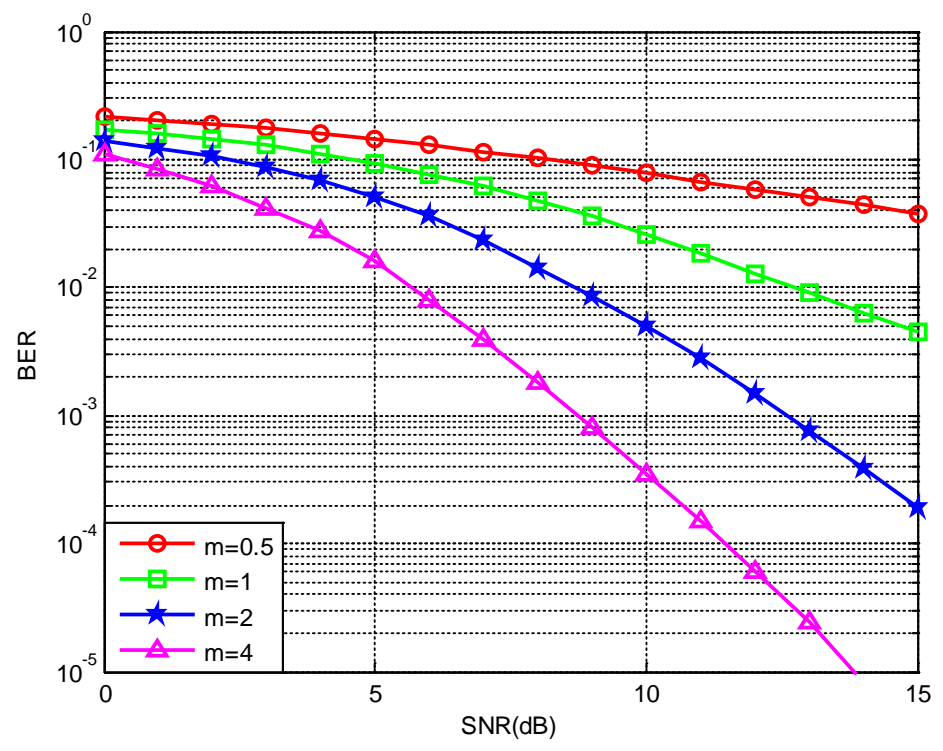

Fig. 2. The BER performances for different shape factors

Table 2. Parameter Settings Adopted in the Simulation of LDPC and Polar Coded Systems

\begin{tabular}{cc}
\hline \hline Parameter & Value \\
\hline Code rate (both for Polar codes and LDPC codes) & 0.5 \\
Code length (for Polar codes) & 1024 bit \\
Code length (for LDPC codes) & 1024 bit \\
Characteristic equation of CRC codes & $f(D)=D^{24}+D^{23}+D^{6}+D^{5}+D^{1}$ \\
List size of SCL decoding & $2,4,8,16$ \\
Frame Number & 10000 \\
\hline \hline
\end{tabular}

In section 2.3, three recursive relations are proposed to determine the proper Bhattacharyya parameter for Nakagami-m fading channels. In order to select the best recursive relation for different fading coefficients, a comparison is obtained with SC decoder. The results are shown in Fig. 3. Take $m=2$ as an example: partial information bits selection (the first 256 bits) under three recursive relations are listed in Table 3, where the 0's represent frozen bits and the 1 's represent information bits. Compared with Type 1, the information bit selection changes of Type 2 and Type 3 are highlighted in red font. Fig. 3 reveals that Type 1 has a better performance among these three types of recursive relations for all the fading coefficients chosen. Therefore, Type 1 recursive relation will be used in the simulations next. 
Table 3. Information Bit Selection under Three Recursive Relations

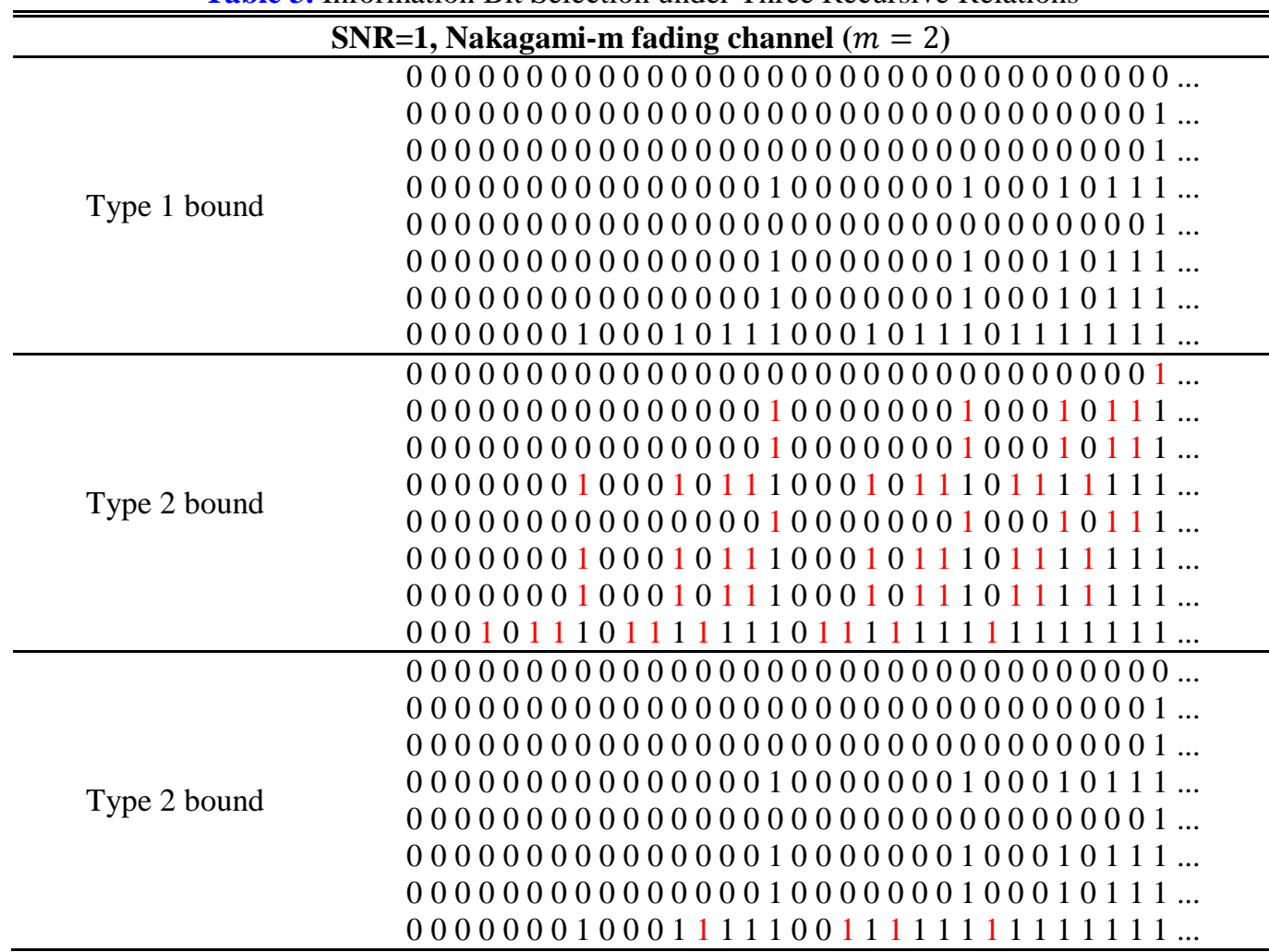

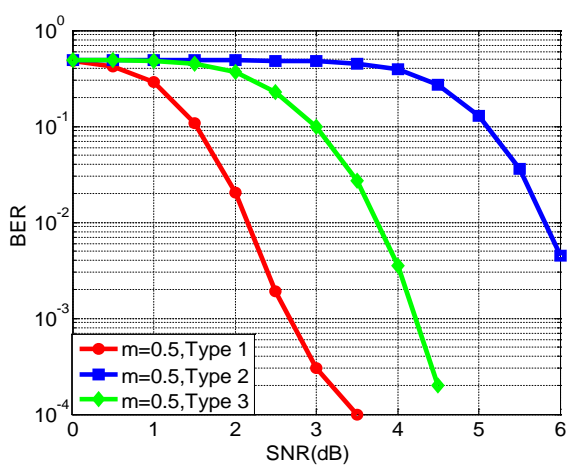

(a) $m=0.5$

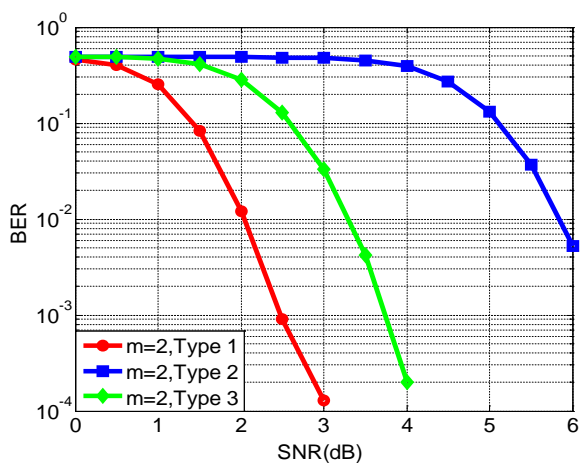

(c) $m=2$

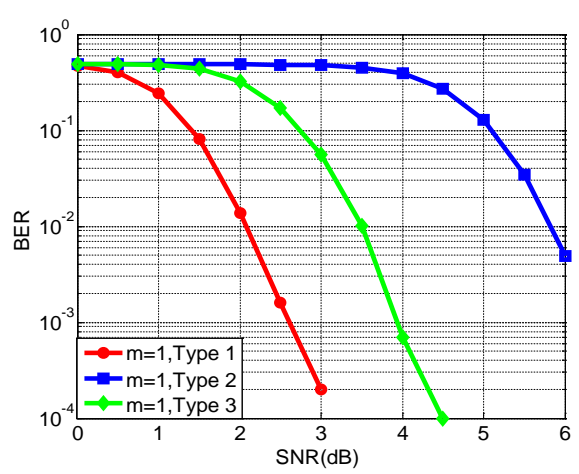

(b) $m=1$

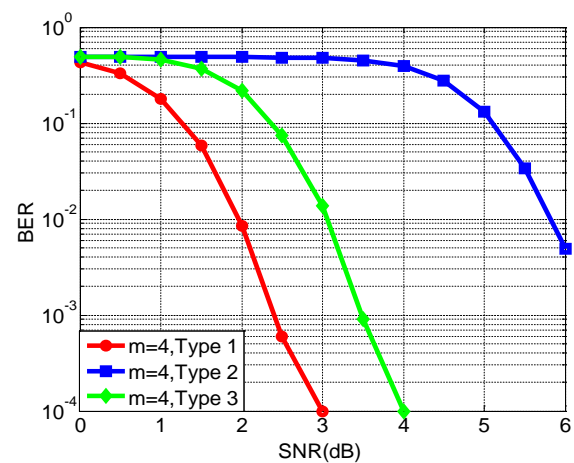

(d) $m=4$

Fig. 3. The performance of three calculation schemes of Bhattacharyya parameters for different values of $m$ 
The performance of OFDM system with and without channel coding over Nakagami-m $(m=2)$ fading channels are simulated here, and the comparison results are shown in Fig. 4. It can be observed that in the smaller SNR range from 0 to $1.5 \mathrm{~dB}$, the performance of the systems with polar codes are worse than that with non-code. The reason is that the signal-to-noise ratio is so low that the error rate of the channel coding is relatively high, so the error correction will be counterproductive. But the average BER performance of OFDM systems have been greatly improved with polar codes at higher SNR region. For example, the coding gain of polar coded OFDM with SCL decoding $(L=2)$ is about $10.1 \mathrm{~dB}$ more than uncoded OFDM as average BER $\approx 10^{-3}$. Furthermore, CRC aided SCL decoding algorithm can effectively reduce the BER of system, which can get other $0.5 \mathrm{~dB}$ gain as compared with SCL decoding algorithm under the same list size as average $B E R \approx 10^{-4}$. Besides, the performance of polar coded with CRC aided SCL $(L=8)$ decoding is obviously better than that of LDPC coded system, and can get about $1.1 \mathrm{~dB}$ gain as average BER $\approx 10^{-5}$.

Based on the simulation of Fig.4, Fig. 5 studies the performance comparison between polar codes and LDPC codes when the code length is longer. In these simulations, we choose the code length of 1024, 2048 and 4096. The results show that, even if the code length is longer, the performance of polar codes is still better than LDPC codes in OFDM system.

In order to achieve ultra-high data transmission rate, high-order modulation schemes will be used in this wireless system [28][29].The simulations of polar coded OFDM systems over Nakagami-m fading channel $(m=2)$ with 256QAM are carried out here, and the results are shown in Fig. 6. Obviously, 256QAM modulation leads to worse average BER than 4QAM modulation.

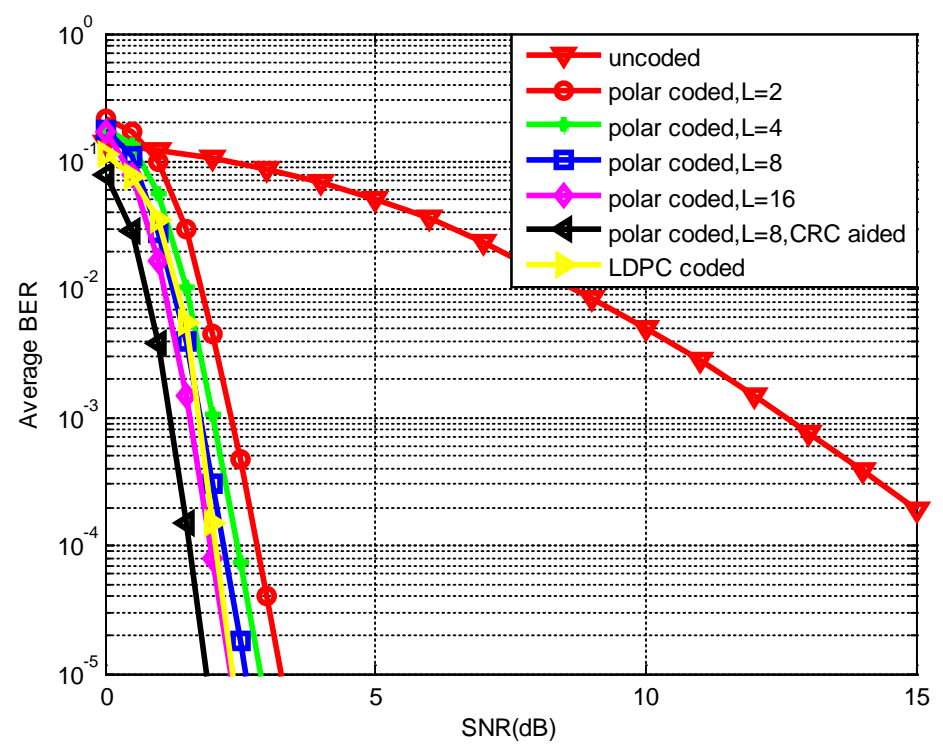

Fig. 4. The average BER performances of OFDM systems with 4QAM modulation 


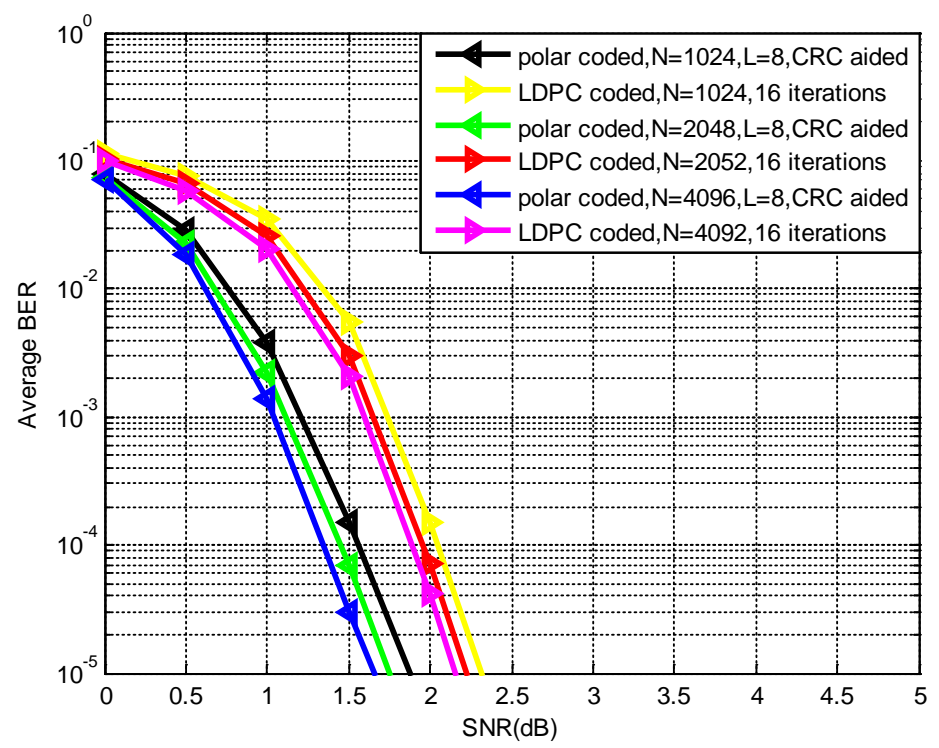

Fig. 5. The average BER performances of OFDM systems for different code length

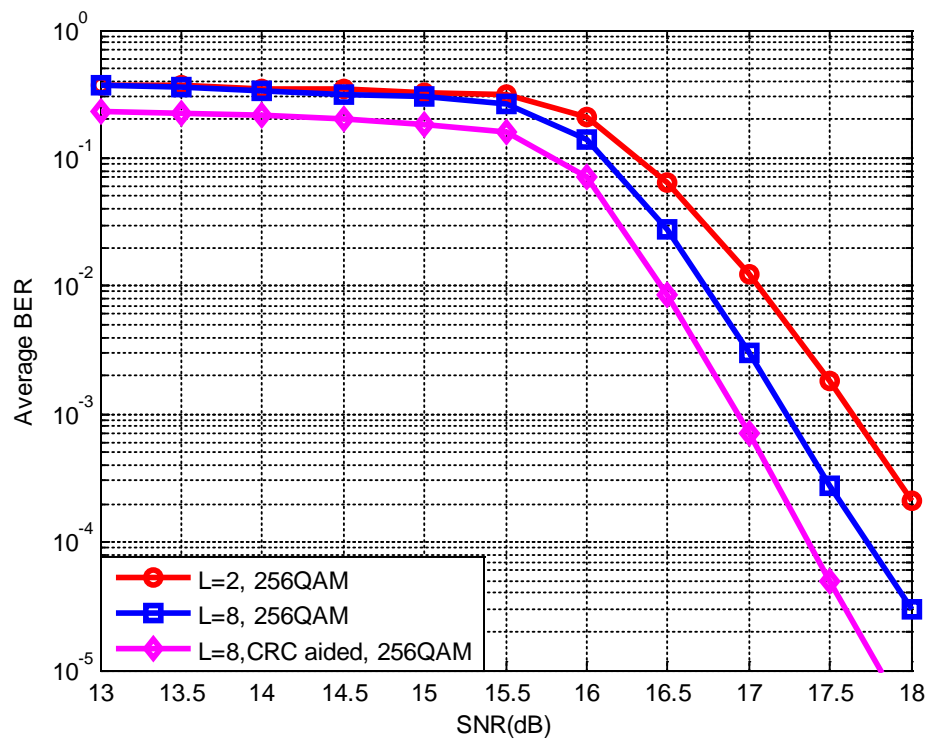

Fig. 6. The average BER performances of polar coded OFDM systems with 256QAM modulation

\section{Conclusion}

In this paper, a OFDM system over Nakagami-m fading channel based on polar code has been studied in detail. A design scheme of polar code for Nakagami-m fading channel based on Bhattacharyya parameter is proposed. According to the simulation results, we can find that the performance of the designed polar codes in Nakagami-m fading channel is good, and it can improve the BER performance of OFDM system. Furthermore, polar coded OFDM system with CRC aided SCL decoding outperforms LDPC coded OFDM system actually. 


\section{References}

[1] S. Huang and Z. Zhang, "Principles of FECs with evaluating different types of FEC used in the Internet and wireless networks," in Proc. of 2011 International Conference on Electronics, Communications and Control (ICECC), Ningbo, China, pp. 2181-2184, 2011. Article (CrossRef Link)

[2] L. L. Hanzo, T. Liew, B. Yeap, R. Tee, and S. X. Ng, Turbo coding, turbo equalisation and space-time coding, Piscataway, Wiley-IEEE press, NJ, USA, 2011. Article (CrossRef Link)

[3] E. Arikan, "Channel Polarization: A Method for Constructing Capacity-Achieving Codes for Symmetric Binary-Input Memoryless Channels,” IEEE Transactions on Information Theory,vol. 55, no. 7, pp. 3051-3073, July 2009. Article (CrossRef Link)

[4] D. Zhou, K. Niu, and C. Dong, “Construction of Polar Codes in Rayleigh Fading Channel,” IEEE Communications Letters, vol. 23, no. 3, pp. 402-405, 1 Mar. 1, 2019. Article (CrossRef Link)

[5] A. Bekkali, C. B. Naila, K. Kazaura, K. Wakamori, and M. Matsumoto, "Transmission Analysis of OFDM-Based Wireless Services Over Turbulent Radio-on-FSO Links Modeled by Gamma Gamma Distribution,” IEEE Photonics Journal, vol. 2, no. 3, pp. 510-520, June. 2010. Article (CrossRef Link)

[6] W. Wu, B. Wang, Y. Zeng, H. Zhang, Z. Yang and Z. Deng, "Robust Secure Beamforming for Wireless Powered Full-Duplex Systems With Self-Energy Recycling,” IEEE Transactions on Vehicular Technology, vol. 66, no. 11, pp. 10055-10069, Nov. 2017. Article (CrossRef Link)

[7] N. J. Borkar and D. S. Bormane, "BER performance of OFDM system with adaptive modulation," in Proc. of 2012 IEEE International Conference on Complex Systems (ICCS), Agadir, Morocco, pp. 1-8, 2012. Article (CrossRef Link)

[8] K. S. Priyanjali and B. S. Ramanjaneyulu, "Performance of MC-CDMA system with various orthogonal spreading codes in multipath Rayleigh fading channel," in Proc. of 2017 IEEE International Conference on Smart Technologies and Management for Computing, Communication, Controls, Energy and Materials (ICSTM), Chennai, India, 309-312, 2017. Article (CrossRef Link)

[9] A. Ramzan and M. O. B. Saeed, "LDPC-coded OFDM-system with BPSK modulation: Performance comparison with uncoded OFDM system," in Proc. of 2018 3rd International Conference on Control and Robotics Engineering (ICCRE), Nagoya, Japan, pp. 213-217, 2018. Article (CrossRef Link)

[10] Rao, G. Rajeswara, and G. S. Rao, "TURBO coded OFDM performance analysis for digital video broadcasting," in Proc. of 2017 International Conference on Trends in Electronics and Informatics (ICEI), Tirunelveli, India, pp. 558-560, 2017. Article (CrossRef Link)

[11] Agarwal, Arun, and S. K. Patra, "Performance prediction of OFDM based Digital Audio Broadcasting system using channel protection mechanisms,” in Proc. of 2011 3rd International Conference on Electronics Computer Technology, Kanyakumari, India, pp. 57-61, 2011. Article (CrossRef Link)

[12] R. Umar, F. Yang and S. Mughal, "BER performance of a polar coded OFDM over different channel models," in Proc. of 2018 15th International Bhurban Conference on Applied Sciences and Technology (IBCAST), Islamabad, Pakistan, pp. 764-769, 2018. Article (CrossRef Link)

[13] A. Mousa and H. Mahmoud, "Channels estimation in OFDM system over rician fading channel based on comb-type pilots arrangement,” IET Signal Processing, vol. 4, no. 5, pp. 598-602, Oct. 2010. Article (CrossRef Link)

[14] W. R. Braun and U. Dersch, “A physical mobile radio channel model,” IEEE Transactions on Vehicular Technology, vol. 40, no. 2, pp. 472-482, May. 1991. Article (CrossRef Link)

[15] V. A. Aalo, C. Mukasa, and G. P. Efthymoglou, "Effect of Mobility on the Outage and BER Performances of Digital Transmissions over Nakagami-m Fading Channels,” IEEE Transactions on Vehicular Technology, vol. 65, no. 4, pp. 2715-2721, April. 2016. Article (CrossRef Link)

[16] Y. Kim, "Starvation Analysis of CDF-Based Scheduling Over Nakagami-m Markov Fading Channels,” IEEE Communications Letters, vol. 22, no. 2, pp. 372-375, Feb. 2018. Article (CrossRef Link) 
[17] I. Tal and A. Vardy, "How to construct polar codes," IEEE Transactions on Information Theory, vol. 59, no. 10, pp. 6562-6582, October 2013. Article (CrossRef Link)

[18] R. Mori and T. Tanaka, "Performance of polar codes with the construction using density evolution," IEEE Communication Letters, vol. 13, no. 7, pp. 519-521, July 2009. Article (CrossRef Link)

[19] Y. Zhang, A. Liu, K. Pan, C. Gong, and S. Yang, “A practical construction method for polar codes,” IEEE Communication Letters, vol. 18, no. 11, pp. 1871-1874, November 2014. Article (CrossRef Link)

[20] D. Wu, Y. Li, and Y. Sun, “ Construction and block error rate analysis of polar codes over awgn channel based on gaussian approximation,” IEEE Communication Letters, vol. 18, no. 7, pp. 1099-1102, July 2014. Article (CrossRef Link)

[21] G. Bonik, S. Goreinov, and N. Zamarashkin, “Construction and analysis of polar and concatenated polar codes: practical approach, ” arXiv, pp. 1-17, July 2012. Article (CrossRef Link)

[22] Vangala, Harish, E. Viterbo, and Y. Hong, "A Comparative Study of Polar Code Constructions for the AWGN Channel," arXiv, Jan. 2015. Article (CrossRef Link)

[23] P. Shi, Wenjuan Tang, S. Zhao and B. Wang, "Performance of polar codes on wireless communication channels," in Proc. of 2012 IEEE 14th International Conference on Communication Technology, Chengdu, China, pp. 1134-1138, 2012. Article (CrossRef Link)

[24] A. Bravo-Santos, "Polar Codes for the Rayleigh Fading Channel,” IEEE Communications Letters, vol. 17, no. 12, pp. 2352-2355, December 2013. Article (CrossRef Link)

[25] I. Tal and A. Vardy, "List Decoding of Polar Codes," IEEE Transactions on Information Theory, vol. 61, no. 5, pp. 2213-2226, May 2015. Article (CrossRef Link)

[26] K. Niu and K. Chen, “CRC-Aided Decoding of Polar Codes,” IEEE Communications Letters, vol. 16, no. 10, pp. 1668-1671, October 2012. Article (CrossRef Link)

[27] S. D. Dissanayake, and J. Armstrong, "Comparison of ACO-OFDM, DCO-OFDM and ADO-OFDM in IM/DD Systems,” Journal of Lightwave Technology, vol. 31, no. 7, pp. 1063-1072, April1, 2013. Article (CrossRef Link)

[28] 3GPP TSG R1-162216, “Candidate NR modulation schemes,” Qualcomm Incorporated, Busan, Korea, Apr. 2016. Article (CrossRef Link)

[29] K. Wu, J. He, J. Ma, and Y. Wei, “A BIPCM Scheme Based on OCT Precoding for a 256-QAM OFDM-VLC System,” IEEE Photonics Technology Letters, vol. 30, no. 21, pp. 1866-1869, 1 Nov.1, 2018. Article (CrossRef Link)

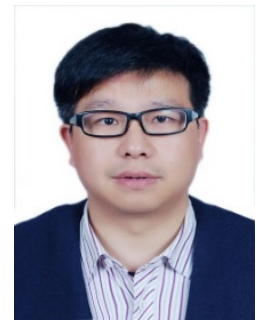

Guo Rui received the Ph.D. degree from the Zhejiang University, Hangzhou, China, in 2007. He is currently an associate professor with the School of Communication Engineering, Hangzhou Dianzi University, Hangzhou, China. He is a visiting scholar at Oregon State University from August 2018 to August 2019. His research interests include wireless communication and channel coding.

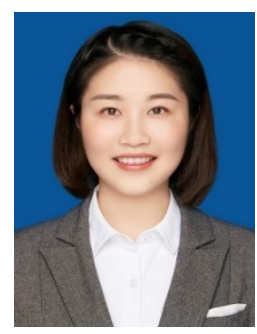

Wu Yingjie is currently pursuing a master's degree at the School of Communication Engineering, Hangzhou Dianzi University. The author's current research interests include wireless communication and channel encoding. 